\title{
Major Stressors and Coping Strategies of Frontline Nursing Staff During the Outbreak of Coronavirus Disease 2020 (COVID-19) in Alabama
}

This article was published in the following Dove Press journal:

Journal of Multidisciplinary Healthcare

\author{
Haneen Ali (iD) ${ }^{\prime}$ \\ Astin Cole ${ }^{2}$ \\ Abdulaziz Ahmed ${ }^{3}$ \\ Sa'd Hamasha ${ }^{4}$ \\ Gabriella Panos ${ }^{5}$ \\ ' Health Services Administration \\ Program, Auburn University, Auburn, AL, \\ USA; ${ }^{2}$ Public Administration, Auburn \\ University, Auburn, AL, USA; ${ }^{3}$ Business \\ Department, University of Minnesota \\ Crookston, Crookston, MN, USA; \\ ${ }^{4}$ Department of Industrial and Systems \\ Engineering, Auburn University, Auburn, \\ AL, USA; ${ }^{5}$ Health Services \\ Administration Program, Auburn \\ University, Auburn, AL, USA
}

Background: Studies suggest that nursing staff during pandemics such as H1N1 Influenza and COVID-19 exhibit higher than usual stress levels due to an increasingly overburdened healthcare system and increasing infection rates. This study aims to investigate the major stressors and coping strategies reported by nurses working directly with potentially infectious patients in Alabama, United States, during the COVID-19 pandemic.

Materials and Methods: A cross-sectional questionnaire study was conducted with nursing staff working in hospital settings in the state. The questionnaire was completed by 109 nurses working in hospitals that treated COVID-19 patients.

Results: Around $71 \%$ of the nursing staff were concerned about receiving more COVID-19 patients and exhibited heightened workload-related stress resulting from taking care of infected patients. The study found that most nurses $(82 \%)$ are stressed about getting their friends and family infected. Overall, younger, less experienced nurses reported more stress levels compared to older, senior-level nurses. Findings suggest that many nurses fail to perceive protective measures as an effective coping strategy, with only $75 \%$ reporting problem-solving strategies such as hand washing and wearing a face mask, and only $60 \%$ avoiding public transportation and crowded spaces. Findings also suggest a lack of organizational support including psychiatric assistance, with no nurses reportedly seeking psychological therapy.

Conclusion: The COVID-19 pandemic increased the stress level of the nursing staff in Alabama. The study finds that the cases in the state of AL are still increasing dramatically, which can overwhelme the healthcare system and escalate nurse stress levels.

Keywords: pandemic, COVID-19, nurses stress, workload, coping strategies, infection

\section{Background}

On December 31st, 2019, the Wuhan Municipal Health Commission reported a novel coronavirus disease (COVID-19) after studying a cluster of cases of pneumonia in Wuhan, China's Hubei Province. ${ }^{1}$ Initial studies reported that the virus spread through social interactions with friends and family. This process resulted in the disease rapidly spreading throughout China. By January 13, 2020, cases of COVID-19 were reported in Thailand, marking its first cases of infection outside of China. ${ }^{1}$ By March 11, 2020, COVID-19 was declared a pandemic by the World Health Organization (WHO), with over 120,000 cases and 4300 deaths
Department of Industrial and Systems Engineering and Health Services Administration Program, Auburn University, 35I W Thach Concourse, 7080 Haley

Center, Auburn, AL 36849, USA

Email hba0007@auburn.edu
Journal of Multidisciplinary Healthcare 2020:13 2057-2068

2057 
reported around the world. ${ }^{2}$ WHO reports that as of October 22, 2020, there are over 40 million confirmed cases worldwide, with over 1 million deaths. ${ }^{2}$

\section{COVID-19}

Coronavirus disease 2019 (COVID-19) is a new disease caused by SARS-CoV-2, a novel coronavirus that humans have not previously seen. The initial data suggests that COVID-19 might be at least five times more lethal than seasonal influenza. ${ }^{3}$ Common symptoms of COVID-19 include nausea, vomiting, shortness of breath, chest pain, dry cough, and fever. ${ }^{1,4}$ Initial reports of the virus suggest that aging adults and people with autoimmune disorders are the most impacted by this disease; however, COVID19 has been found to impact all groups of people. ${ }^{1,3-9}$

WHO reports that the United States (US) has 8,184,788 confirmed cases of COVID-19 infection, and over 200,000 deaths as of October. ${ }^{2}$ Due to the widespread infection rate and the already high ratio of patients to personnel in ICUs, many news reports, politicians, and public health officials are using the capacity of the ICU and other emergency health functions as the litmus test for healthcare shortages. ${ }^{10-13}$

A scoping review seeking to estimate a global number of healthcare workers (HCW) infected or deceased due to the pandemic found 152,888 infections and 1413 deaths reported. ${ }^{7}$ Frontline nurses, due to their daily routine consisting of monitoring and physically assisting multiple severely infectious patients, may be more susceptible to the psychosocial hazards of working with patients with COVID-19. ${ }^{14-24}$ According to the World Health Organization (WHO), not only are healthcare workers at a higher risk of infection, their transmission of the virus during their time as asymptomatic individuals can amplify the severity of the infection in their healthcare system. ${ }^{25}$ In the situation report, WHO cites additional hazards for HCWs during this time such as higher workload, psychological distress, fatigue, occupational burnout, and physical and psychological violence. ${ }^{25}$

In addition to the poor outcomes caused by the loss of productivity, reports suggest that rising infection rates can cause an overwhelming burden on hospital infrastructure. 26,27 One study found that $20 \%$ of patients hospitalized with COVID-19 in a New York hospital were admitted to the ICU and required mechanical ventilation. ${ }^{26}$ Another study of 21 critically ill patients in Washington State, USA, found that $81 \%$ of pneumonia patients with COVID-19 were admitted to the ICU, with $71 \%$ of these patients requiring mechanical ventilation. ${ }^{27 \text { These studies sug- }}$ gest that the burden on hospital capacity is largely placed on the emergency and intensive care units.26,27

\section{Nursing Staff Stress: An Ethical Dilemma}

The American Nurses Association's (ANA) Code of Ethics for Nurses with Interpretive Statements (2015) is perceived by most as the nursing profession's standard of best ethical practices in the United States. ${ }^{28}$ While provision 2 of their code of ethics states that "the nurse's primary commitment is to the patient," provision 5 of the code states that "the nurse owes the same duty to themselves as to others. ${ }^{28}$ Nursing ethics at the international level create similar confusion regarding the moral implications of nursing during a pandemic. The International Council of Nurses (ICN) International Code of Ethics states that within nursing there is a respect for human rights related to equality in patient healthcare treatment that may feel threatened or at times compromised due to resource shortages felt by ICUs filled overcapacity. ${ }^{29}$ Turale et al. (2020) provides insights through the experience of one notable emergency room nurse, Melissa Farmer, reporting how nurses in their hospital working 60 hours a week with bruises on their noses from wearing face masks, being informed by physicians about the "difficult decisions that will be made in the coming weeks about whom to resuscitate" due to a lack of ventilators. ${ }^{30}$ On top of all of this, nursing staff during this time must also screen phone calls from the anxious family members of patients infected with COVID-19 who are not allowed into the hospital to comfort their loved one due to the need to socially distance. ${ }^{30}$ While both provisions are regarded as non-negotiable ethical standards within their communities, these obligations are found to cause additional jobrelated burdens on nurse staff during pandemics when nurses must work under extreme conditions to continually care for critically ill infected patients. . $^{14,22,24,28,29,31,32}$

Due to professional obligations to provide patients with the best treatment possible, many nurses may feel the ethical strain of watching patients die due to a lack of ventilators or suffer anxiety from the risk of infecting patients as asymptomatic carriers. ${ }^{19-21,28,31,33}$ Further, nurses struggling to balance their well-being with their sense of professional tasks, ${ }^{34,35}$ or nurses who must work during a pandemic due to financial limitations, ${ }^{36,37}$ may feel additional emotional and psychological effects. From an ethical perspective, nurses may experience moral injury or trauma as they must balance their perceived 
responsibility to safeguard patient welfare with the fear and anxiety of risking infection and the spread of the infection outside of the hospital setting. ${ }^{31,32}$

\section{Adverse Effects of Nursing Staff Stress}

The struggle of nurses to maintain their mental and emotional health while providing care to severely ill and infectious patients can create a professionally and ethically compromising position that some nurses are unable to effectively cope with. ${ }^{31,33,38}$ These sentiments have been reported in the news as factors for adverse events such as recent nurse suicides reported in the $\mathrm{US}^{39}$ and Italy. ${ }^{33}$ An ICU nurse from West Palm Beach, Florida hospital was found dead from a drug overdose in his car before his shift at work, with his friends and family heavily suggesting that heavy drug use was a coping mechanism for the long shift hours and high emotional stress of watching infectious patients die. ${ }^{39}$ In Europe, the National Federation of Italian Nurses reported that an ICU nurse in San Gerardo hospital at Monza, near Milan, committed suicide after contracting COVID-19. ${ }^{33}$ The National Federation of Italian Nurse report suggests that the nurse committed suicide due to the fear and guilt felt at the idea that she may have amplified the spread of infection in their hospital as an asymptomatic carrier. ${ }^{33}$ Previous studies during the 2009 H1N1 Influenza pandemic have shown that nurses suffered from psychological effects due to stress during the epidemic. ${ }^{40,41}$ While the transmission method, infection rate, and mortality rate may vary amongst infections, many notable pandemics such as the 2009 H1N1 influenza have been found to overburden emergency and intensive care functions across states. ${ }^{40,41}$ Further, continuously high levels of stress and long work hours have been linked with substance abuse, ${ }^{38}$ as well as increased instances of depression $^{42}$ and suicide. $^{37}$

\section{Investigating Nurse Stressors in Alabama}

Madison County reported the first case of COVID-19 in the state of Alabama on March 17th. ${ }^{43}$ According to the Centers for Disease Control and Prevention (CDC) COVID-19 Case Surveillance Public Use Data, as of November, Alabama was reported to have 195,000 confirmed cases and 2973 deaths. $^{10}$ As the number of COVID-19 cases in Alabama is expected to increase as the infection rate rises, the study expects to see exceptionally greater burdens on the state's hospital infrastructure. Further, the study expects to see numerous negative outcomes such as greater emotional and mental workload for healthcare professionals working during COVID-19.14,16,22,24 To better understand these negative externalities, additional research is required that investigates the severity of the social, emotional, and mental impact of working as a nurse during the COVID-19 pandemic.

While studies have investigated nurse stress during both the current pandemic ${ }^{14,19,20,22,24}$ as well as previous outbreaks, ${ }^{16,44}$ there are still gaps in the discussion regarding how nurse staff perceives the efficacy of psychological intervention as a coping strategy. For example, Cai et al. (2020) in studying nurse stress during the COVID-19 pandemic note the importance of psychosocial resources such as therapy provided by in-house psychologists. ${ }^{14}$ Overall, the study suggests the importance of connecting hospital resources to nurse stress, even during a pandemic. However, such studies largely assume the prevalence of organizational support such as psychosocial resources, with other studies observing that nurse staff may not even perceive the resource as beneficial to them, opting out of using it altogether. ${ }^{45}$

Research is also required to understand the extent to which individual characteristics such as age, seniority, being married, or having children affects perceived stress levels for nurse staff working during the COVID-19 pandemic. In more recent work, Babore et al. (2020), as well as Labrague \& de los Santos (2020), found that working with patients infected with COVID-19 increased stress levels investigated nurse stress during COVID-19. ${ }^{22,24}$ Interestingly, the studies found that individual characteristics were not significant in explaining variations in stress levels. These findings run counter to many observations which suggest that being young, single, and less experienced is associated with greater stress levels among nurse staff. $^{20,46}$ As a result, further testing is required to further examine the psychological impact and coping strategies of nurses while working under the COVID-19 pandemic.

\section{Objectives}

The study aims at capturing nurse staff's perceived stressors to determine what factors contribute to the highest levels of stress along demographic dimensions, as well as which coping strategies provide the most consistent form of stress reduction for nurses during a pandemic in the state of Alabama, United States.

\section{Materials and Methods}

A cross-sectional survey study was conducted in the state of Alabama. The study was approved by Auburn 
University's Institutional Review Board (IRB reference \# is: 20-238 EX 2005). All participants provided informed consent to participate, and that this study was conducted in accordance with the Declaration of Helsinki. Participants were notified about the aims of the project and the risks that might be associated with the survey study on the first page of the survey. Additionally, participants were notified that no identifiable information will be collected, their participation is voluntary and by completing the survey, they are agreeing to participate in the study. Informed consent was provided at the beginning of each survey, and participants were compensated for their time if they choose to continue and take the survey (\$20).

Nursing staff who are working during the pandemic in hospital settings were invited to participate in a survey that was shared and verified using LinkedIn. ${ }^{22}$ The PI and COPIs shared the Qualtrics link on their LinkedIn with a short introduction about the survey. A total of 126 participants responded to the survey between May 19th and June 6th of 2020. Twelve participants did not complete the survey, and five participants were verified by Qualtrics as out of the state nurses. The data for 109 surveys were analyzed.

\section{Questionnaire Development}

Principal items were drawn based on the questionnaire designed by Lee et al (2005) that was used to identify the sources of stress and coping strategies during the 2003 SARS epidemic and used again by Cai et al (2020) to evaluate similar factors and mental health outcomes among nurses in Hubei, China during the COVID-19 pandemic, and other existing instruments in the literature. ${ }^{14,16,20,21,44,47}$ A preliminary list of potential nurse stressors and coping strategies was created and distributed to the first group of experts (2 ICU nurses, one general nurse, one clinical nursing faculty, and one public health expert). An online focus group session was conducted with the authors and the first group to discuss the preliminary list of items. As a result of the experts' comments, five items were added, three were removed, and the wording of fifteen items were revised. A second item list was prepared and distributed to the second group of experts [one nursing faculty, three nurses (1 ICU, 1 General nurse, 1 ER), and one nursing manager], and they were requested to vote individually on keeping or discarding each item. A focus group session was then conducted with both groups and the authors to solve the disagreements.
The survey instrument consisted of four components: a demographic and work-related data component (nine items), a stressor-scale component [comprised of five constructs: Stress from taking care of patients (three items), Stress from assignments and workload (six items), Stress from personal life, friends, and colleagues (five items), Stress from a lack of knowledge about Covid-19 (nine items), Stress from the environment (six items)], nurses feelings component (open-ended question), and coping strategies component [comprised of three constructs: Avoidance (four items), Problemsolving (four items), Transference (four items), and one open-ended question to investigate other coping strategies]. The stressor items were measured with a 5-point Likert scale ranging from 1 (not at all) to 5 (very much). Stress coping strategies were investigated by counting the number of coping strategies employed by nurses that were grouped into Avoidance, Problem-solving, and Transference, respectively. Participants were asked to mark all the coping strategies they employed and the score of each category was calculated based on the count of recorded strategies (e $\mathrm{g}$ the category was given a score of 1 if the participant is not employing any coping strategy within this category. If the participant is employing the 4 coping strategies, the category was given a score of 5).

\section{Measures Tested}

\section{Stressors}

Stress with Working with Patients Infected with COVID-19 (DI)

The questions corresponding with this variable, (reported as Domain 1 or "D1") are used to measure to what degree nurses experienced stress in working with patients infected with COVID-19. ${ }^{9,14,26,46,48}$

\section{Stress Caused by Additional Workload and Assignment} (D2)

Due to the increased workload caused by providing emotional and medical care for highly infectious patients, the study expects to see nurses reporting additional stress as they receive more COVID-19 cases. The questions corresponding with this variable (D2) are used to measure to what degree workload associated with COVID-19 contributed to nursing stress. ${ }^{14,16}$

Stressed Caused by Personal Life, Friends, and Colleagues (D3) 
Many frontline nurses are likely scared of getting their friends and family infected. ${ }^{14,16}$ The questions corresponding with this factor (D3) are used to measure to what degree these fears and concerns contributed to nurses' stress.

Stress Due to Lack of Knowledge About the COVID-19 (D4)

Information regarding the transmission, impacted demographics, and severity of COVID-19 is either highly limited or has changed greatly since initial reporting in March. ${ }^{2,10}$ As a result, the study expects to see nurses reporting increased levels of stress caused by the uncertainty of changing policies related to COVID-19. ${ }^{14,16}$ The questions corresponding with this factor (D4) are used to measure to what degree the uncertainty caused by a lack of reliable information contributed to nurse stress.

\section{Stress from the Environment (D5)}

The initial studies suggest that constant screening, shortage in PPE and ventilators, and shortage of staff contributed to a higher stress level among nurses. ${ }^{14,22,24}$ The questions corresponding with this factor (D5) are used to measure to what degree nurses perceived their environment as a source of stress.

\section{Coping Strategies}

Avoidance (CDI)

Avoidance in this study refers to actions taken by nurses to avoid physical exposure to any stimuli related to the pandemic. ${ }^{14,18,22,38,49-51}$ The study will use the Avoidance variable (identified as coping domain 1 or "CD1") to measure to what degree nurses relied on avoidance as a coping strategy.

\section{Problem-Solving (CD2)}

Problem-solving in this study (CD2) refers to all professional steps nurses take to reduce their stress by reducing their risk of infection. ${ }^{14,18,22,38,49-51}$ The study will be using the problem-solving variable to measure to what degree nurses relied on problem-solving as a coping mechanism.

\section{Transference (CD3)}

Transference here is defined as the redirection of feelings and thoughts about one person onto another person. Transference is broken into four lists of items that ask respondents on their preferred choice of transference such as religions, friends, and professional help such as psychiatric. ${ }^{14,49,50}$ While transference has been used to consider "passive" occurrences such as differences of perspectives due to switching languages among bilingual people. ${ }^{52}$ In this case, we operationalize transference from the perspective of nurse staff wanting to transfer their negative feelings about their role as frontline healthcare workers to professionals in psychological programs, caring members of religious organizations, or concerned family members. ${ }^{14,49,50} \mathrm{We}$ find this measure to not only help capture the role of social distancing due to the pandemic in limiting interpersonal communication, but it also provides insights into the preferences of nurses as to whom they want to transfer their negative feelings, (or even whether they want to transfer them at all). The study will be using the transference variable (CD3) to measure to what degree nurses relied on transference as a coping strategy.

\section{Reliability and Validity}

Reliability was tested by the means of internal consistency, which reflects the inter-relatedness among the items in each construct Cronbach's alpha, with a value $>0.70$ being considered acceptable. ${ }^{53}$ The pilot survey was distributed to 10 nurses in Auburn, AL, and Cronbach's alpha ranged from $0.74-0.89$. Test-retest stability overtime was not tested. Content validity was assessed based on the feedback and input from the two groups in the instrument design stage.

\section{Statistical Analysis}

Data from completed questionnaires were processed and analyzed using Minitab 19. Descriptive statistics such as mean value, standard deviation, frequency, and mean stress constructs were used to describe participant characteristics and domains. Multiple linear regression analysis was used to examine the relationship between the demographic information and the stressors domains as the study determined the data was normally distributed. Chi-squared $\chi 2$ test was used to compare the responses to coping strategies employed by the nursing staff between different demographic groups.

\section{Results}

The demographic and work-related information for 109 surveys were analyzed. Table 1 shows that $89 \%(n=97)$ of the participants were female. The participants were relatively young, with $40 \%(n=44)$ under 30 years old. The diversity of the participants was relatively narrow, 
Table I Characteristics of the Study Participants/Demographic Information

\begin{tabular}{|c|c|c|c|}
\hline Demographic Information & $\mathbf{N}$ & $\begin{array}{l}\text { Std. } \\
\text { Deviation }\end{array}$ & $\begin{array}{l}\text { Frequency } \\
\%\end{array}$ \\
\hline Age & 109 & 1.214 & \\
\hline$<30$ & 44 & & 40.45 \\
\hline $31-40$ & 22 & & 20.2 \\
\hline $4 I-50$ & 17 & & 15.6 \\
\hline$>50$ & 26 & & 23.9 \\
\hline Gender & 109 & 1.89 & \\
\hline Female & 97 & & 89 \\
\hline Male & 12 & & 11 \\
\hline Ethnicity & 109 & 1.1 & \\
\hline White & 98 & & 89.9 \\
\hline African American & 11 & & 10.1 \\
\hline Others & & & \\
\hline Marital status & & 2.87 & \\
\hline Married & 53 & & 48.6 \\
\hline Divorced & 10 & & 9.2 \\
\hline Never married & 46 & & 42.2 \\
\hline Parent status & 109 & 1.35 & \\
\hline No & 71 & & 65.1 \\
\hline Yes & 38 & & 34.9 \\
\hline Seniority & 109 & 1.6 & \\
\hline Primary & 53 & & 48.6 \\
\hline Intermediate & 47 & & 43.1 \\
\hline Senior & 9 & & 8.3 \\
\hline Specialty & 109 & 3.48 & \\
\hline General nurse & 33 & & 30.3 \\
\hline $\mathrm{ICU}$ & 31 & & 28.4 \\
\hline OR & 19 & & 17.4 \\
\hline ER & 12 & & 11 \\
\hline Other & 14 & & 12.8 \\
\hline Shift & 109 & 1.33 & 100 \\
\hline Morning & 73 & & 66.4 \\
\hline Night & 36 & & 32.7 \\
\hline \multicolumn{4}{|l|}{ Number of hospitalized } \\
\hline \multicolumn{4}{|l|}{ COVID-19 patients } \\
\hline $\mathrm{I}=<100$ & 21 & 0.957 & 19.3 \\
\hline $2=101-250$ & 34 & & 31.2 \\
\hline $3=25 I-400$ & 39 & & 35.8 \\
\hline $4 \leq 40$ I & 15 & & 13.8 \\
\hline
\end{tabular}

with the majority of the participants identifying as White Americans $(90 \%)(\mathrm{n}=98)$. Relationship status was relatively even at around $50 \%(\mathrm{n}=54)$ of the participants reporting they were married. Only $35 \%(n=38)$ of nurses reported having children. The majority of respondents had limited work experience, with only approximately $9 \%(n=$ 10) of the participants reported having a senior-level position. Additionally, 28\% $(n=31)$ were ICU nurses. Most of the participants worked during the morning shift $(66.4 \%)(n=72)$ and around $14 \%(n=15)$ worked in hospitals that had hospitalization of more than 350 COVID-19 patients.

\section{Stressor Domains}

\section{Stress from Taking Care of Patients (DI)}

The results from the multiple regression show that the number of patients with COVID admitted to the hospital has a significant correlation with $\mathrm{D} 1(\mathrm{p}=0.004)$. In addition, parental status $(p=0.004)$, seniority $(p=0.007)$, and specialty $(\mathrm{p}=0.000)$ were all found to have a significant effect on the level of D1. In addition, 68.6\% ( $\mathrm{n}=74)$ of nurses reported concerns about their patients' condition getting worse, and $63 \%(n=69)$ were worried about communication with patients and their families. On the domain level, around $72 \%(n=79)$ of the younger nurses reported higher stress resulting from the condition of their COVID19 patients getting worse. A significant difference was also found in parental status where single nurses and nurses without children reported having higher stress levels. Senior nurses reported lower stress levels, ICU and ER nurses reported higher stress levels.

\section{Stress from Assignments and Workload (D2)}

The results show that $70 \%(\mathrm{n}=76)$ of nurses are concerned about getting more infected patients and $70.5 \%(\mathrm{n}=77)$ are concerned about having to wear face masks for a long number of hours. On the domain level, statistically significant differences were found in relationship status $(\mathrm{p}=$ $0.000)$ ) where single nurses reported more stress, and seniority $(p=0.000)$ were senior nurses reporting less stress.

\section{Stress from Personal Life, Friends, and Colleagues (D3)}

The results show that around $74 \%(n=81)$ of nursing staff are worried about getting infected, and $70 \%$ are worried about colleagues getting infected. Further, $82 \%(n=89)$ of nurses are worried that their families will get infected, with $65 \%(\mathrm{n}=71)$ reported being concerned about being avoided by their friends and family members. On the domain level, statistically significant differences were found in the number of admitted patients with COVID$19(\mathrm{p}=0.000)$ which was linked to a higher stress level, relationship status $(p=0.001)$ with single nurses reporting 
more stress, seniority $(\mathrm{p}=0.002)$ with senior nurses reporting less stress, and specialty $(p=0.003)$ with ICU nurses reporting more stress.

\section{Stress from a Lack of Knowledge About Covid-19 (D4)}

Results from the descriptive analysis show that $77 \%(n=84)$ of participants were concerned with the lack of specific treatment for COVID-19, with $76 \%(n=83)$ of nurses concerned with a lack of psychological support for patients. Additionally, more than $76 \%(n=83)$ of nurses were concerned with constant changes to regulations related to COVID-19, with $63 \%$ $(n=69)$ concerned about the stay at home order being lifted too early and around $79 \%(n=86)$ were concerned about how long the pandemic will last. Further, $65 \%(n=71)$ were stressed about hearing the news/reports for the number of new cases every day, and $62 \%(n=68)$ was stressed about being constantly screened for infection.

On the domain level, the number of patients with COVID admitted to the hospital was statistically significant and linked to a higher level of stress $(p=0.039)$. Gender was also found to be statistically significant $(\mathrm{p}=$ 0.000 ) with female participants reporting more stress. Both relationship status $(\mathrm{p}=0.018)$ and parental status $(\mathrm{p}=$ 0.009 ) were found to be statistically significant, with single participants and participants without children reporting less stress. Nurse specialty was also found to be statistically significant, $(\mathrm{p}=0.000)$ with participants working in operating rooms, pediatric units, and maternity units reporting less concern and number of patients.

\section{Stress from the Environment (D5)}

The results show that around $65 \%(n=71)$ of nurses were concerned about the lack of psychological support from their healthcare organization. Around $77 \%(n=84)$ of nurses reported stress when they hear news related to hospital staff shortages, around 79\% $(\mathrm{n}=86)$ are worried about PPE shortages, and $69 \%(n=75)$ were worried about ventilator shortages and $79 \%(n=86)$ are concerned about face masks shortage. On the domain level, statistically significant differences were found in gender $(\mathrm{p}=0.000)$, with female nurses reporting higher stress. Relationship status $(p=0.006)$ was found to be statistically significant, with single participants reporting higher stressors. Seniority was found to be statistically significant $(\mathrm{p}<0.003)$ with senior-level nurses reporting less stress. Lastly, specialty $(\mathrm{p}=0.00)$ was found to be statistically significant with ICU nurses reporting higher stress.

\section{Nurses Feelings During the Pandemic (Open-Ended Question)}

Nurses responded to the open-ended question; the words they used are summarized in Table 2. Fatigue was found to be the most prevalent word (95 times) used to summarize hospital nurses' feelings about their experience in working due to the COVID-19 pandemic, with anxiety (76) and burnout tied for 2nd place. Other high scoring emotions (used more than 60 times) included stress, sadness, and frustration in descending order. While fatigue is highly common and expected for nurses engaging in infectious patients, the high use of particularly impactful words such as anxiety, burnout, and depression leave the investigators wondering why nurses are not using or reporting the use of psychological therapy.

\section{Coping Strategies}

\section{Avoidance (CDI)}

The results show that around $36 \%(n=39)$ express that they are avoiding working overtime to reduce exposure to COVID-19 patients. Around $10 \%(n=11)$ of nurses reported avoiding work with COVID-19 patients, 50.4\% $(n=55)$ reported avoiding media news about COVID-19 and related

Table 2 Nurses Response to Open-Ended Questions

\begin{tabular}{|l|l|l|}
\hline Words Used & $\begin{array}{l}\text { Number of } \\
\text { Times } \\
\text { Mentioned }\end{array}$ & $\%$ \\
\hline Sadness & 68 & 62.38532 \\
\hline Stress & 71 & 65.13761 \\
\hline Anxiety & 76 & 69.72477 \\
\hline Fatigue & 95 & 87.15596 \\
\hline Burned out & 76 & 69.72477 \\
\hline Fear & 35 & 32.11009 \\
\hline Depression & 64 & 58.7156 \\
\hline Emotional & 47 & 43.11927 \\
\hline Exhaustion & 43 & 39.44954 \\
\hline Discomfort & 26 & 23.85321 \\
\hline Frustration & 73 & 66.97248 \\
\hline $\begin{array}{l}\text { Upset/depressed/Anger toward } \\
\text { unsupportive management }\end{array}$ & 8 & 7.33945 \\
\hline $\begin{array}{l}\text { Do not feel seeing people or } \\
\text { friends }\end{array}$ & 5 & 3.669725 \\
\hline Guilt & 4 & \\
\hline
\end{tabular}


information such as infection and mortality rates. Around $6 \%(\mathrm{n}=7)$ of nurses reported avoiding seeing people in general. On the domain level, there were statistically significant differences in the Avoidance domain among age $(\chi 2$ $=24.8, \mathrm{p}<0.001, d f=3)$ and specialty $\left(\chi^{2}=20.57, \mathrm{p}<\right.$ $0.001, d f=4)$, with younger nurses and OR, pediatric, and maternity nurses reporting more use of avoidance strategies.

\section{Problem-Solving (CD2)}

The results show that around $75 \%(n=82)$ of nurses are following strict protective measures (handwashing, face masks, protective gear, etc.), with $37 \%(n=40)$ reported learning about COVID-19, its prevention, and mechanism of transmission. Around $57 \%(\mathrm{n}=62.13)$ reported practicing social distancing to reduce infection, with $51 \%(\mathrm{n}=56)$ reportedly taking necessary precautions when grocery shopping. On the domain-level, there were statistically significant differences in the problem-solving domain among gender $(\chi 2$ $=21.252, \mathrm{p}<0.001, d f=1)$ with male nurses reporting less use of problem-solving strategies. Further, relationship status $(\chi 2=6.5, \mathrm{p}<0.05, d f=2)$ and parental status $(\chi 2=7.184, \mathrm{p}=$ $0.007, d f=1$ ) were found to be statistically significant, with single participants and participants without children reporting the higher levels. Lastly, specialty $(\chi 2=9.912, p<0.05$, $d f=4$ ) was found to be statistically significant, with general nurses and ICU nurses reporting higher use.

\section{Transference (CD3)}

The results found that $41.2 \%(n=45)$ of nurses are practicing religious or spiritual activities such as prayer, meditation, and reading. Around $73 \%(\mathrm{n}=80)$ of nurses are practicing relaxation activities in their free time, such as watching movies, reading, exercising, etc. Further, around $73 \%(\mathrm{n}=80)$ reported chatting with family and friends to relieve stress and obtain support. The results found that no nurses reported ever seeking help from a psychologist as a coping strategy. On the domain level, there were statistically significant differences in the number of hospitalized COVID-19 patients $(\chi 2=12.920, \mathrm{p} \leq 0.005, d f=3)$, where nurses working in hospitals with fewer admitted patients reported the highest score for using transference.

\section{Other Coping Strategies (CD4)}

The results show that over $10 \%$ of nurses were engaged in coping strategies not listed above. Seven nurses (6.3\%) reported spending time with their children as a coping strategy during the pandemic, with $4.6 \%$ reporting engaging in general activities with their children, and 1.8\% reporting the use of arts and crafts with their children.
Further, 6 nurses (5.5\%) reported drinking alcohol as a coping strategy. None of these coping strategies were found to be statistically significant.

\section{Discussion}

This study finds that nurses are experiencing additional emotional and mental stress during the current outbreak. The study shows that nurses exhibited workload-related stress resulting from taking care of infected patients and stress resulting from receiving more patients, with $71 \%$ of nurses are concerned about receiving more COVID-19 cases. These findings are inconsistent with Cai et al. (2020), whose findings suggest that nurse commitment and a sense of responsibility in ensuring healthcare delivery suppressed fears and anxiety in working with patients with COVID-19. ${ }^{14}$ However, Lee (2005) studying previous pandemics such as the $2004 \mathrm{H} 1 \mathrm{~N} 1$ influenza pandemic found similar results, citing factors such as stress, fatigue, and burnout as nurses have to spend several days in a row looking after highly infectious patients. ${ }^{16}$ This study also found that most hospital nurses (82\%) were stressed about getting their friends and family infected. This is consistent with both Lee et al. (2005) and Cai et al. (2020), who found that nurses are afraid of spreading their heightened risk of infection to their loved ones. ${ }^{14,16}$

This study's findings suggest that professional experience may play a significant role in the way nurses perceive stress during a pandemic. On one hand, a relatively new nurse may perceive stressors as more emotionally or mentally impactful due to a lack of experience in dealing with various levels of patient acuity, resource capacity, or supervision. On the other hand, a senior nurse may have developed greater emotional and mental resilience out of job necessity, suggesting that nurses learn how to process certain stressors as less impactful over time. These findings support evidence regarding the relationship between seniority and age on job stress in healthcare systems. ${ }^{20,21,46,51}$

\section{Differences in Stress Levels and Stress-Related Outcomes on Demographic Levels}

While differences along experience levels can explain trends related to differences in stress within various age groups, others such as nurse concerns with hospital administrative outcomes (eg the amount of PPE, the prevalence of staff shortages), and the number of COVID-19 cases lend to other explanations. Even though younger, less 
experienced nurses reported higher stress as a result of COVID-19, more than 52\% $(n=57)$ of respondents considered quitting their job as a result of the COVID-19 pandemic. Further, because most respondents were over the age of 40 , the study finds that nurses are likely experiencing stress beyond the level that additional experience can overcome. This study's findings provide insights that scholars suggested were absent from this pandemic, as certain works found no differences in anxiety and stress levels due to individual characteristics. ${ }^{24}$ For example, it was found that $68.6 \%(n=74)$ of nurses reported concerns about their patients' condition getting worse, with $72 \%$ $(n=79)$ of the younger nurses reporting higher stress overall. These findings highly suggest that job stressors such as patient acuity as well as individual characteristics such as age play crucial roles in the way frontline nurse staff perceive stress levels. Further, $63 \%(n=69)$ were worried about communication with patients and their families. Individual characteristics may provide a moderating effect on the stress levels of nurse staff engaging with highly infectious patients with rapidly deteriorating health in many cases. ${ }^{30}$ Further, age may also contribute to anxiety and stress levels perceived by nurse staff in completing tasks requiring high amounts of emotional fortitude such as screening phone calls from the anxious family members of patients infected with COVID-19. ${ }^{30}$ This study's findings provide further evidence that such job demand stressors related to frontline nurse staff may be tied to the ethical challenges found in taking care of infectious patients while also taking care of yourself or loved ones. ${ }^{31,32}$ Additionally, this study's findings provide contextual support for the moral injury and trauma sustained by frontline nurse staff who choose to continue treating patients infected with COVID-19 despite their feelings of fear and burnout. ${ }^{32}$ Further research is needed to explore the motivations of frontline nurse staff, and there capacity to influence salient human resource variables. ${ }^{54,55}$

\section{Differences in Risk Perception}

This study's findings suggest that there is a difference in the perspective of nurses as professionals versus nurses as parents. Related studies have found similar insights that can be found from studies observing the health decisions of healthcare workers during previous pandemics. For example, one survey study capturing the perspectives of healthcare workers with children during the 2009 H1N1 influenza pandemic found that $\mathrm{HCW}$ uses the rationale of a patient rather than that of a professional to make their health decisions, regarding preventative measures for the pandemic. ${ }^{41}$ These findings suggest that nurses may perceive the efficacy of healthcare treatments and procedures differently when making health decisions for themselves and as a parent. Further, given the timing and availability of risk information, the study also supports the idea that HCWs are subject to the same or similar biases related to health information outside of their expertise. In the case of COVID-19, this study finds that many nurses in Alabama are likely making decisions for themselves and their children based on information provided by media coverage of COVID-19, which has emphasized the disease's impact on aging patients. This study's findings support literature suggesting that hospital nurse parents think like average civilians rather than professional health care workers outside of work. ${ }^{41}$ Further, the findings support studies suggesting that HCWs with lower health literacy may fail to perceive the efficacy of healthcare delivery. ${ }^{41,56-58}$

\section{Differences in Coping Strategies}

It was suggested by literature that avoidance strategies are an attempt to avoid unnecessary interactions or new COVID-19 information might be a way for nurses to escape from stressors caused by overly engaging. ${ }^{49,50}$ These nurses may seek to only engage with patients and the relevant information in so far as to ensure professional accountability. This study found that many participants $(50 \%, \mathrm{n}=54)$ reported avoiding media coverage providing updates on COVID-19 infection and mortality statistics. While the media coverage may not provide a direct benefit to hospital nurses, updated reports on infection and mortality rates are often accompanied by reports of additional infection sites designated "hot spot" zones. Studies on pandemic health outcomes and risk communication found the prevalence of media in the dissemination of information related as significant in understanding the health and safety habits of HCW during previous pandemics such as the 2009 H1N1 influenza. ${ }^{41}$ Following this logic, this study's findings may suggest that a reliance on the avoidance strategy for nurses could significantly limit their access to updated risk information which may include improved studies or additional protective measures.

Additionally, this study finds that protective measures are not readily used by all nurses as coping strategies, with only $75 \%(n=82)$ of nurses reporting that they follow strict protective measures such as hand-washing, face masks, protective gear, etc. to reduce their risk of infection. Despite all nurses knowing to help prevent the spread 
of COVID-19, up to $25 \%(\mathrm{n}=27)$ failed to perceive protective measures as their most effective strategy to reduce their risk of COVID-19, their risk of getting others infected, and the overall burden to their hospital's capacity. The difference among certain groups of nurses in adopting protective measures may be indicative of differences in their perception of the severity and characteristics of COVID-19 based on the limited information provided by media. This study's findings are ultimately consistent with Babore (2020) and other works finding problem-solving as the dominant and most significant coping strategy in reducing frontline nurse staff stress. ${ }^{14,18,22,49,51}$

\section{Differences in Findings: A Lack of Organizational Support}

Cai et al. (2020) and Lee et al. (2005) found that nurses' experiences during the COVID-19 and 2003 SARS pandemic had both positive and negative psychological impacts on nurses. ${ }^{14,16}$ For example, while many felt nervous and worried, the study found that many nurses reported feeling their service was an "act of duty". ${ }^{16}$ The authors suggest that while worrying about infecting their families and colleagues, nurses were able to cope with the situation through various means. It was found that the importance of hospital administration provided psychological support as a crucial factor in the nurse's ability to overcome their challenges. ${ }^{14-16,20,23}$ Lee et al. (2005) report that a psychiatric team including two senior psychiatrists and two psychologists provided various psychiatric services including psychoeducation debriefing groups, a counseling hotline, and individual psychotherapy. ${ }^{16}$ Further, Cai et al. (2020) cite current psychological assistance services provided in China and recommends further assistance to support the psychological and emotional burdens of nursing. The study recommends a tailored approach similar to Cai et al. (2020), whereby frontline nurse staff are encouraged to speak with a loved one through video chat or receive individual psychotherapy at least once during their shift. ${ }^{14}$ Literature suggests that due to the fear of getting their families infected, nurse staff may not be spending as much time engaging with friends and family. ${ }^{14,24}$ This creates a gap in social support that can be addressed through compassionate management policies.

\section{Limitations}

While this study found that frontline nurse staff experience heightened stress from the pandemic, the current design is limited in its capacity to make causal inferences outside of associations and trends within hypothesized relationships between job demands and individual characteristics and nurse staff stress. Additionally, the nurses sampled largely came from the two dominant nonprofit hospitals in the state. ${ }^{13,43}$ However, the insights should not be understated, as the results reflect regional variations within the state of Alabama, and as a result, provide insights into variations of nurse staff stress that would otherwise be more difficult to capture with aggregate data from the general population.

\section{Conclusion}

The study shows that the COVID-19 pandemic causes an increase in the stress level for nurses in Alabama, with nurses exhibiting workload-related stress resulting from taking care of infected patients and stress resulting from receiving more patients. Further, the study finds the concerns raised in the survey by nurses related to hospital resources and the lack of reported usage regarding psychological therapy as a transference coping strategy lend some evidence of organizational gaps whereby access to the service might be limited (or non-existent). This study's findings ultimately support literature raising awareness of the importance of health literacy in risk communication, ${ }^{41,56-58}$ as well as literature seeking to further understand the sources of stress among frontline nurse staff. ${ }^{14,16,19,20,22-24,51}$

\section{Acknowledgment}

The authors would like to thank Auburn University for supporting this research project. The study was funded through Dr. Ali's (corresponding author) start-up funds.

\section{Disclosure}

The authors have no conflicts of interest to disclose.

\section{References}

1. Mackenzie JS, Smith DW. COVID-19: a novel zoonotic disease caused by a coronavirus from China: what we know and what we don't. Microbiol Aust. 2020;41(1):45. doi:10.1071/MA20013

2. WHO. World Health Organization Information Dashboard. 2020.

3. Faust JS, Del Rio C. Assessment of deaths from COVID-19 and from seasonal influenza. JAMA Intern Med. 2020;180(8):1045. doi:10.1001/ jamainternmed.2020.2306

4. Holshue ML, DeBolt C, Lindquist S, et al. First case of 2019 novel coronavirus in the United States. $N$ Engl J Med. 2020;382 (10):929-936. doi:10.1056/NEJMoa2001191

5. Fang DM Social-Cultural, Religious, and Health System Barriers to Hepatitis B Screening Among Hmong: A Case Study [ProQuest Dissertations and Theses]. 2016. 
6. Kuehn BM. New insights on COVID-19's hyperinflammation in children. JAMA. 2020;324(15):1489. doi:10.1001/jama.2020.20151

7. Bandyopadhyay S, Baticulon R, Kadhum $M$, et al. Infection and mortality of healthcare workers worldwide from COVID-19: a scoping review. medRxiv. 2020. doi:10.1101/2020.06.04.20119594

8. Gu T, Mack JA, Salvatore M, et al. Characteristics associated with racial/ethnic disparities in COVID-19 outcomes in an academic health care system. JAMA Netw Open. 2020;3(10):e2025197e2025197. doi:10.1001/jamanetworkopen.2020.25197

9. Yancy CW. COVID-19 and African Americans. JAMA. 2020;323 (19):1891-1892. doi:10.1001/jama.2020.6548

10. Alabama's COVID-19 risk indicator dashboard; 2020. Available from: https://alpublichealth.maps.arcgis.com/apps/opsdashboard/ index.html. Accessed November 2, 2020.

11. Cohen L. Washington state to require face masks after county runs out of hospital beds; 2020. Available from: https://www.cbsnews. $\mathrm{com} /$ news/washington-state-require-face-mask-hospital-bedscoronavirus/. Accessed February 6, 2020

12. Giannakeas V, Bhatia D, Warkentin MT, Bogoch II, Stall NM. Estimating the maximum capacity of COVID-19 cases manageable per day given a health care system's constrained resources. Ann Intern Med. 2020;173(5):407-410. doi:10.7326/M20-1169

13. Walsh L. Alabama health officials worry about hospital capacity as COVID-19 cases rise; 2020. Available from: https://abc3340.com/ news/local/alabama-health-officials-worry-about-hospital-capacity-as -covid-19-cases-rise. Accessed February 6, 2020.

14. Cai H, Tu B, Ma J, et al. Psychological impacts and coping strategies of front-line medical staff during COVID-19 outbreak in Hunan, China. Med Sci Monit. 2020;26. doi:10.12659/MSM.924171

15. Kang L, Ma S, Chen M, et al. Impact on mental health and perceptions of psychological care among medical and nursing staff in Wuhan during the 2019 novel coronavirus disease outbreak: a cross-sectional study. Brain Behav Immun. 2020;87:11-17. doi:10.1016/j.bbi.2020.03.028

16. Lee S-H, Juang -Y-Y, Su Y-J, Lee H-L, Lin Y-H, Chao CC. Facing SARS: psychological impacts on SARS team nurses and psychiatric services in a Taiwan general hospital. Gen Hosp Psychiatry. 2005;27 (5):352-358. doi:10.1016/j.genhosppsych.2005.04.007

17. Kniffin KM, Narayanan J, Anseel F, et al. COVID-19 and the workplace: implications, issues, and insights for future research and action. Am Psychol. 2020. doi:10.1037/amp0000716

18. Shechter A, Diaz F, Moise N, et al. Psychological distress, coping behaviors, and preferences for support among New York healthcare workers during the COVID-19 pandemic. Gen Hosp Psychiatry. 2020;66:1-8. doi:10.1016/j.genhosppsych.2020.06.007

19. Bohlken J, Schömig F, Lemke MR, Pumberger M, Riedel-Heller SG. COVID-19 pandemic: stress experience of healthcare workers: a short current review. Psychiatr Prax. 2020;47(4). doi:10.1055/a-1159-5551

20. Shen X, Zou X, Zhong X, Yan J, Li L. Psychological stress of ICU nurses in the time of COVID-19. Crit Care. 2020;24(1). doi:10.1186/s13054-020-02926-2

21. Kalichman SC, Gueritault-Chalvin V, Demi A. Sources of occupational stress and coping strategies among nurses working in AIDS care. $J$ Assoc Nurses AIDS Care. 2000;11(3):31-37. doi:10.1016/S1055-3290(06)60274-4

22. Babore A, Lombardi L, Viceconti ML, et al. Psychological effects of the COVID-2019 pandemic: perceived stress and coping strategies among healthcare professionals. Psychiatry Res. 2020;293:113366. doi:10.1016/ j.psychres.2020.113366

23. Vagni M, Maiorano T, Giostra V, Pajardi D. Hardiness, stress and secondary trauma in Italian healthcare and emergency workers during the COVID-19 pandemic. Sustainability. 2020;12(14). doi:10.3390/ su12145592

24. Labrague LJ, Santos JAA. COVID-19 anxiety among front-line nurses: predictive role of organisational support, personal resilience and social support. $J$ Nurs Manag. 2020;28(7):1653-1661. doi:10.1111/jonm.13121
25. WHO. Novel Coronavirus (2019-Ncov) Situation Report. 2020.

26. Richardson S, Hirsch JS, Narasimhan M, et al. Presenting characteristics, comorbidities, and outcomes among 5700 patients hospitalized with COVID-19 in the New York city area. JAMA. 2020;323 (20):2052. doi:10.1001/jama.2020.6775

27. Arentz M, Yim E, Klaff L, et al. Characteristics and outcomes of 21 critically ill patients with COVID-19 in Washington state. JAMA. 2020;323(16):1612. doi:10.1001/jama.2020.4326

28. American Nurses Association. Ethics for Nurses with Interpretive Statements. 2015.

29. International Council of Nurses. The ICN Code of Ethics for Nurses. 2012.

30. Turale S, Meechamnan C, Kunaviktikul W. Challenging times: ethics, nursing and the COVID-19 pandemic. Int Nurs Rev. 2020;67 (2):164-167. doi:10.1111/inr.12598

31. Aliakbari F, Hammad K, Bahrami M, Aein F. Ethical and legal challenges associated with disaster nursing. Nurs Ethics. 2015;22 (4):493-503. doi:10.1177/0969733014534877

32. Stovall M, Hansen L, van Ryn M. A critical review: moral injury in nurses in the aftermath of a patient safety incident. $J$ Nurs Scholarsh. 2020;52(3):320-328. doi:10.1111/jnu.12551

33. Rahman A, Plummer V. COVID-19 related suicide among hospital nurses; case study evidence from worldwide media reports. Psychiatry Res. 2020;291:113272. doi:10.1016/j. psychres.2020.113272

34. Nantsupawat A, Nantsupawat R, Kunaviktikul W, Turale S, Poghosyan L. Nurse burnout, nurse-reported quality of care, and patient outcomes in Thai Hospitals. J Nurs Scholarsh. 2016;48 (1):83-90. doi:10.1111/jnu.12187

35. Thomas CM, Bantz DL, McIntosh CE. Nurse faculty burnout and strategies to avoid it. Teach Learn Nurs. 2019;14(2):111-116. doi:10.1016/j.teln.2018.12.005

36. Prapanjaroensin A, Patrician PA, Vance DE. Conservation of resources theory in nurse burnout and patient safety. $J$ Adv Nurs. 2017;73(11):2558-2565. doi:10.1111/jan.13348

37. Davidson JE, Proudfoot J, Lee K, Terterian G, Zisook S. A longitudinal analysis of nurse suicide in the United States (2005-2016) with recommendations for action. Worldviews Evid Based Nurs. 2020;17(1):6-15. doi:10.1111/wvn.12419

38. Rafati F, Nouhi E, Sabzevari S, Dehghan-Nayeri N. Coping strategies of nursing students for dealing with stress in clinical setting: a qualitative study. Electron Physician. 2017;9(12):6120-6128. doi:10.19082/6120

39. Garcia-Navarro L. Florida COVID ICU nurse remembered by his father; 2020. Available from: https://www.npr.org/2020/05/24/ 861630493/florida-covid-icu-nurse-remembered-by-his-father. Accessed February 6, 2020.

40. Goulia P, Mantas C, Dimitroula D, Mantis D, Hyphantis T. General hospital staff worries, perceived sufficiency of information and associated psychological distress during the $\mathrm{A} / \mathrm{H} 1 \mathrm{~N} 1$ influenza pandemic. BMC Infect Dis. 2010;10(1). doi:10.1186/1471-2334-10-322

41. Torun SD, Torun F, Catak B. Healthcare workers as parents: attitudes toward vaccinating their children against pandemic influenza A/ H1N1. BMC Public Health. 2010;10(1). doi:10.1186/1471-2458-10596

42. Pappa S, Ntella V, Giannakas T, Giannakoulis VG, Papoutsi E, Katsaounou P. Prevalence of depression, anxiety, and insomnia among healthcare workers during the COVID-19 pandemic: a systematic review and meta-analysis. Brain Behav Immun. 2020;88:901-907. doi:10.1016/j. bbi.2020.05.026

43. Landers KM. First COVID-19 case confirmed in Madison county: Alabama department of public health (ADPH). Available from: http:// www.alabamapublichealth.gov/news/2020/03/17.html. Accessed February 6, 2020.

44. Honey M, Wang WY. New Zealand nurses perceptions of caring for patients with influenza A (H1N1). Nurs Crit Care. 2013;18(2):63-69. doi:10.1111/j.1478-5153.2012.00520.x 
45. Bridges J. Covid-19: supporting nurses' psychological and mental health. J Clin Nurs. 2020;29(15-16). doi:10.1111/jocn.15307

46. Ali H, Cole A, Sienkiewicz A, Ho T. Perspectives of nursing homes staff on the nature of residents-initiated call lights. SAGE Open Nurs. 2020;6:237796082090354. doi:10.1177/2377960820903546

47. Monticone M, Ferrante S, Giorgi I, Galandra C, Rocca B, Foti C. The 27-item coping strategies questionnaire - revised: confirmatory factor analysis, reliability and validity in italian-speaking subjects with chronic pain. Pain Res Manag. 2014;19(3):956828. doi:10.1155/ 2014/956828

48. Belhadjer Z, Méot M, Bajolle F, et al. Acute heart failure in multisystem inflammatory syndrome in children in the context of global SARS-CoV-2 pandemic. Circulation. 2020;142(5):429-436. doi:10.1161/CIRCULATIONAHA. 120.048360

49. Amirkhan JH. A factor analytically derived measure of coping: the coping strategy indicator. J Pers Soc Psychol. 1990;59(5):1066-1074. doi:10.1037/0022-3514.59.5.1066

50. Eslami Akbar R, Elahi N, Mohammadi E, Fallahi Khoshknab M. What strategies do the nurses apply to cope with job Stress? A qualitative study. Glob J Health Sci. 2015;8(6):55. doi:10.5539/ gjhs.v8n6p55

51. Windarwati HD, Ati NAL, Paraswati MD, et al. Stressor, coping mechanism, and motivation among health care workers in dealing with stress due to the COVID-19 pandemic in Indonesia. Asian J Psychiatr. 2020:102470. doi:10.1016/j.ajp.2020.102470.
52. Foster RP. Psychoanalysis and the Bilingual Patient: Some Observations on the Influence of Language Choice on the Transference. Vol. 9. 1992.

53. Tavakol M, Dennick R. Making sense of Cronbach's alpha. Int J Med Educ. 2011;2:53-55. doi:10.5116/ijme.4dfb.8dfd

54. Juhdi N, Pa'wan F, Hansaram RMK. HR practices and turnover intention: the mediating roles of organizational commitment and organizational engagement in a selected region in Malaysia. Int J Hum Resour Manag. 2013;24(15):3002-3019. doi:10.1080/ 09585192.2013.763841

55. Delery J, Shaw J, Ferris G. Research in Personnel and Human Resources Management. 2001:165-197.

56. Lorini C, Collini F, Gasparini F, et al. Health literacy, vaccine confidence and influenza vaccination uptake among nursing home staff: a cross-sectional study conducted in tuscany. Vaccines. 2020;8(2):154. doi:10.3390/vaccines 8020154

57. Nutbeam D. Health literacy as a public health goal: a challenge for contemporary health education and communication strategies into the 21st century. Health Promot Int. 2000;15(3). doi:10.1093/heapro/ 15.3.259

58. Nutbeam D, Levin-Zamir D, Rowlands G. Health literacy in context. Int J Environ Res Public Health. 2018;15(12):2657. doi:10.3390/ ijerph15122657
Journal of Multidisciplinary Healthcare

\section{Publish your work in this journal}

The Journal of Multidisciplinary Healthcare is an international, peerreviewed open-access journal that aims to represent and publish research in healthcare areas delivered by practitioners of different disciplines. This includes studies and reviews conducted by multidisciplinary teams as well as research which evaluates the results or conduct of such teams or healthcare processes in general. The journal

\section{Dovepress}

covers a very wide range of areas and welcomes submissions from practitioners at all levels, from all over the world. The manuscript management system is completely online and includes a very quick and fair peer-review system. Visit http://www.dovepress.com/testimonials. php to read real quotes from published authors. 